\title{
Personal Assistant to Facilitate User Task Automation
}

\author{
Rasika Anerao ${ }^{1}$, Utkarsh Mehta ${ }^{2}$, Sharangdhar Vaze ${ }^{3}$, G. Hrishikesh ${ }^{4}$ \\ \#1,2,3,4 Pursuing B.E.at Computer Engineering Department of P. E. S. Modern College of Engineering, Shivajinagar, Pune, \\ Maharashtra, India
}

\begin{abstract}
This paper presents the approach to develop a personal assistant that reduces the use of input devices like mouse and keyboard on our PC. Giving commands via speech makes it user friendly. This paper also briefly describes the representation model and the available personal assistants in the market. The additional feature of remote access makes it more usable. Moreover addition of new commands to the system makes it extensible.
\end{abstract}

Keywords- Personal assistant, remote access, addition of new commands

\section{INTRODUCTION}

The Personal Assistant is nothing but an implementation of assistance virtually on the user`s PC (Personal Computer). The software can be used with voice, keyboard input and also using internet for remote access. There are some predefined commands in the system and new commands may be added as and when necessary. System notifies the user about new emails, update changes in social networking sites, news reports, etc. User can set alarms, reminders, appointments etc. Tasks like shutdown, lock system, sleep, file reading and writing can also be executed by the system. There are several modules for each task like time, alarm, search etc. The GUI calls these predefined modules for the given commands. System initiates at startup and voice input to the system can be suspended with a command. All the tasks that require the use of keyboard and/or mouse can be handled by this system.

\section{A. Scope}

Anyone who can speak and hear can use this software. Speech commands are given in a specified format or in normal sentence. The software can be given commands by a remote access portal, most commonly a website. It can alert user when specific event occurs making it intelligent.

\section{B. Mathematical Representation of the system}

\section{Input Set:}

The personal assistant takes input via speech, text or textual input through remote signal. So the set of inputs will be,

I1 $=\{1$ word command, fixed sentence command, random sentences $\}$

$\mathrm{I} 2=\{$ text, speech, remote command $\}$

Thus, I = I1 U I2
Input $\mathrm{I}=\{$ all sentences in English via speech, all sentences in English via text, remote command

\section{Output Set:}

The output for the specified inputs above will be response determined by the system according to the input given and the database containing all the necessary inputs and their respective outputs.

$\mathrm{O} 1=\{$ speech, display, text $\}$

$\mathrm{O} 2=\{$ GUI, application response $\}$

Thus, $\mathrm{O}=\mathrm{O} 1 \mathrm{U} \mathrm{O} 2$

Output $\mathrm{O}=\{$ Response for corresponding input via speech, Response for corresponding input via GUI, application response\}

There is a one to one relation between input and output as there is only one output for a particular input.

\section{OPERATIONS}

Following are the operations performed on the input in the system:

1. Recognize (): This operation basically gets the input from the user. For speech input, it converts the speech into text and save it. For text, the input is saved directly while in case of remote signal, the signal is directly converted to the command.

2. Extract (): This operation analyses the input string saved and system gets an idea about which command is expected to be executed for the given input.

3. Search (): This operation searches the local database for the response of the command exacted by the previous operation.

4. Response (): This operation gives the output that we see on the screen or via speech for the given command. 


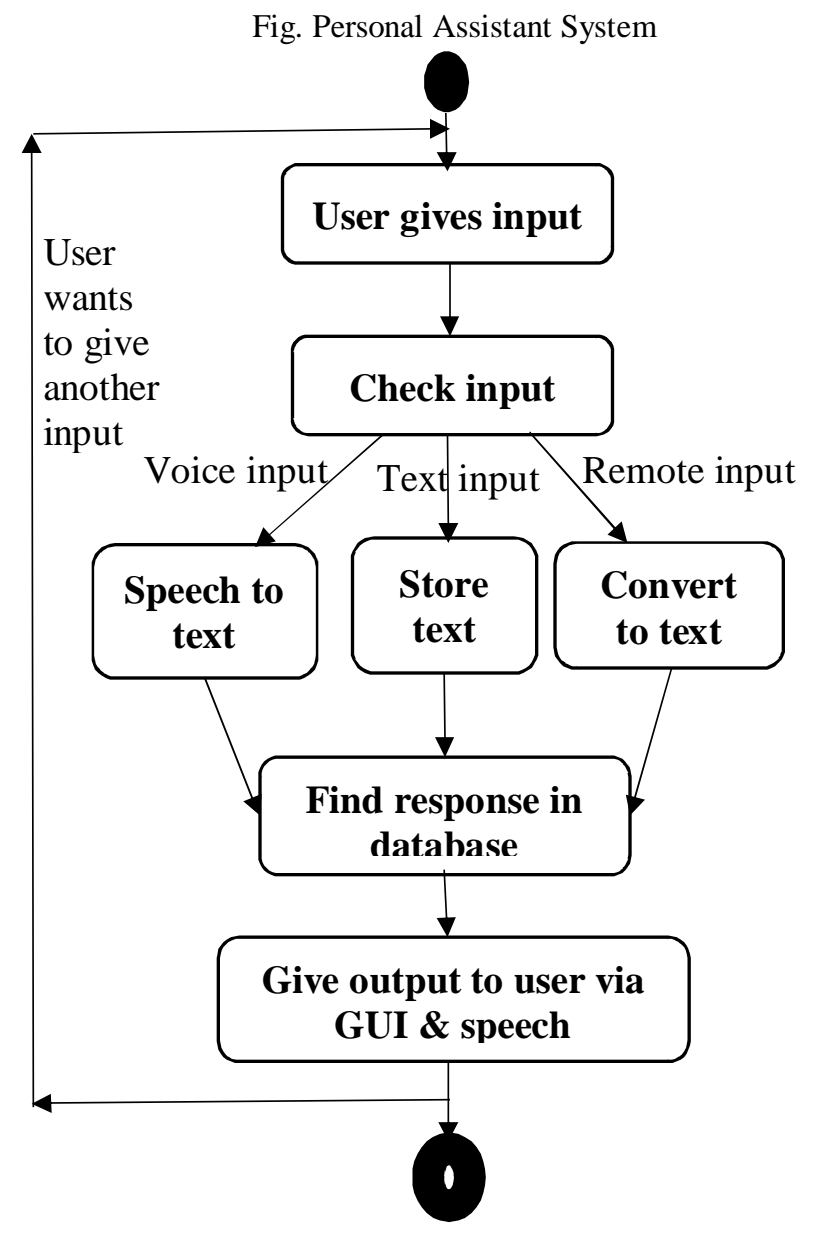

III. LITERATURE SURVEY

Various assistants available in the market include DRAGON - Naturally Speaking, Syn Virtual Assistant, Braina, etc. Also assistants popularly used in the smartphones are Cortana, Siri, and Google now etc.

Dragon - Naturally speaking assistant [1] that is available in the market has the functionalities of dictation, text to speech and command input. Although it needs identical configurations on the computers in the networked environment for assistance. Syn Virtual Assistant [2] available is mainly for voice commands that are used to connect to online services. Facility to add new commands is available but very few commands are predefined here. Braina [3] is an assistant that is developed especially for computers and its tasks. Here an android app is used to interact with the system over a Wi-Fi network. Other popular assistants available are, Google Now [4], Siri [5] and Cortana [6]. Now our goal is to add all these functionalities into a single system making it simpler and user- friendly. Also for the remote access, using a website, commands can be added to the system along with the execution of tasks.

So the summarised list of tasks that can be implemented is as follows:
1. Weather forecast

2. News alerts

3. Alarm

4. Date and time

5. Volume control

6. Opening and closing applications

7. Locks the workstation.

8. Hibernates the system.

9. Power-off the system.

10. Sleep your machine.

11. Birthday remainder

12. Facebook alerts

13. Play typical song

14. Access specific file

15. Dictation

16. Email alert

17. Mail someone directly with speech

18. Use the assistant using mobile app

19. Search nearby places

20. Set reminders

21. Access maps using voice commands

22. Access internet using voice commands

23. Conversation with the assistant

24. Get answers for the questions asked (Assistant uses internet to get the correct answers of the questions asked).

25. Adding new commands (We can add new voice command by providing the action that is supposed to happen when the command is given).

\section{CONCLUSIONS}

This paper introduces with the approach that can used to develop a personal assistant. Though a number of assistants are available in the market for smartphones, personal assistants for PC's are not that popular in our country. Moreover an additional feature of remote access eradicates the limitation of area within which the system can be accessed. Through a site the system can be given commands and this helps to develop an expert system of home automation which is an extended scope of this system.

\section{ACKNOWLEDGMENT}

The authors of this paper would like to thank Ms. Manasi K. Kulkarni and Ms. Manisha C. Petare whose comments and suggestions helped in publishing the paper.

\section{REFERENCES}

[1]Nuance Dragon Assistant http://www.nuance.com/dragon/dragonassistant/index.html

[2]Syn Virtual Assistant http://download.cnet.com/Syn-VirtualAssistant/3000-2346_4-75910908.html

[3]Braina (Brain Artificial) is an intelligent personal assistant software http://www.brainasoft.com/braina/

[4]Google now features http://lifehacker.com/top-10-awesome-features-ofgoogle-now-1577427243

[5]Siri http://en.wikipedia.org/wiki/Siri

[6]Cortana Windows $\quad$ phone 8.1 personal assistant http://www. windowsphone.com/en-us/features-8-1 